\title{
Reconstrucciones desmontables como alternativa reversible en el proceso de reintegración de materiales arqueológicos
}

\author{
Trinidad Pasíes Oviedo
}

Resumen: El tratamiento de lagunas es una de las fases de la restauración que afecta a los diferentes bienes culturales de forma más directa, y muchas son las cuestiones éticas que implica el proceso de reintegración, desde la elección del material a las exigencias de reconocimiento y reversibilidad de estas intervenciones. Las investigaciones que hemos llevado a cabo en el Museo de Prehistoria de Valencia se encaminan a la aplicación de reconstrucciones volumétricas desmontables sobre diferentes materiales de procedencia arqueológica, ya sea cerámica, hueso, vidrio o mosaico, que minimizan los riesgos sobre el original y garantizan su fácil eliminación en el caso de que se considerase necesario.

Palabras clave: Reintegraciones desmontables; Materiales arqueológicos; Reversibilidad.

\section{Reconstruções desmontáveis como alternativa reversível ao processo de reintegração de materiais arqueológicos}

Resumo: $O$ tratamento de lacunas é uma das fases de restauro que mais directamente afeta os diferentes bens culturais e muitas são as questões éticas que o processo de reintegração implica, desde a escolha do material às exigências de reconhecimento e de reversibilidade dessas intervenções. As intervenções que desenvolvemos no Museu de Pré-históra de Valência baseiam-se na aplicação de reconstruções volumétricas desmontáveis, sobre diferentes materiais de origen arqueológica, quer seja cerâmica, osso, vidro ou mosaico, que minimizam os riscos sobre o original e garantem a sua fácil eliminação, no caso de se considerar necessário.

Palavras chave: Reintegrações desmontáveis; Materiais arqueológicos; Reversibilidade.

\section{Demountable reconstructions as an alternative to reversible reintegration process of archaeological} materials

\begin{abstract}
Filling the missing areas is one of the most important phases in the restoration process, which directly affects the cultural objects. This process involves many ethical issues, as the choice of materials or the requirement of recognition and reversibility of these interventions. We have carried out a research in the Museo de Prehistoria de Valencia about the application of detachable restorations on different archaeological materials like pottery, bone, glass or mosaic. This research attempts to minimise damages on the original piece and guarantee an easy removal if it is necessary.
\end{abstract}

Keywords: Detachable restorations; Archaeological materials; Reversibility

\section{Introducción}

Dentro de las operaciones que el conservador-restaurador lleva a cabo en el laboratorio sobre cualquier tipo de bien de interés cultural, una de las que siempre ha generado mayor controversia ha sido la reintegración de lagunas. Normalmente se define como un proceso necesario para facilitar la comprensión de aquellas piezas que han perdido parte de su significado a consecuencia de una alteración. De hecho estas operaciones no se incluyen dentro de una actividad de "conservación", que evita o frena la degradación del objeto, sino que se considera que forman parte de los 
tratamientos de "restauración", que modifican la apariencia del bien con el objetivo de mejorar su lectura (ICOM 2008). Pero lo que no podemos negar es que cualquier decisión que tomemos a la hora de llevar a cabo un proceso de reintegración de lagunas será severamente evaluada, al tratarse del aspecto estético final que tendrá la pieza, convirtiéndose por tanto en una operación de la que a menudo depende el juicio de valor final sobre nuestra intervención.

Sabemos que se caracteriza por ser una actuación directa, cuya polémica no está solo en la elección del método o el producto que se utilizará, sino en los criterios éticos y estéticos que el profesional deberá cumplir. En este último punto son dos los principales requisitos que se deben garantizar: el reconocimiento de los añadidos y la exigencia de reversibilidad. En materiales de procedencia arqueológica se unen, sin embargo, dos conceptos más: la autenticidad y el respeto máximo al original conservado. Este ha sido el principal objetivo de nuestra investigación; buscar alternativas en las que el criterio real de reversibilidad pueda convivir perfectamente dentro de un proceso de reintegración, y que lo haga teniendo como referencia el ideal de la mínima intervención.

\section{Entre la exigencia de la reversibilidad y el criterio de mínima intervención}

El criterio de la reversibilidad aplicado a cualquier proceso de intervención en patrimonio parece universalmente aceptado desde que empezara a ser incluido en los primeros manuales y normativas de la historia de la restauración. Obligatoriamente hemos tenido que incluir este concepto en cualquier propuesta de intervención, informe o publicación que pretenda ser considerada ajustada a criterio. Sin embargo, su significado no está exento de cierta confusión, lo que en los últimos años ha dado lugar a confrontados debates sobre su definición real y la idoneidad del término.

La mayoría de autores que han reflexionado sobre el supuesto de la "reversibilidad" acuerdan que se trata de un concepto abstracto, utópico y que solo se puede contemplar como posibilidad teórica (Oddy 1999; Della Torre 2003: 17). Un término que, además, no puede aplicarse como objetivo único de forma absoluta, ni de igual modo a cualquier proceso de restauración, por lo que a menudo será más conveniente hablar de "solubilidad", "compatibilidad", "posibilidad de re-aplicación" o "eliminación". De hecho cualquier profesional entendería la exigencia de poder remover o hacer soluble un adhesivo empleado para el montaje, por ejemplo, de una pieza cerámica, pero sería más complicado aplicar el concepto de reversibilidad a un proceso de consolidación de un revestimiento de pintura mural, cuando lo que aquí se pretende es aplicar un producto que de cohesión al material que se conserva disgregado y que preferentemente sea compatible con el original. Por otra parte, muchos profesionales apuntan que es incorrecto hablar de "productos reversibles", y que deberíamos emplear en todo caso este concepto para describir los procesos y no los materiales (Appelbaum 1987: 65-66). Y si hablamos entonces de procesos, es en el de la reintegración donde quizá esté más claro el objetivo de "reversible" o "eliminable".

Uno de los requerimientos que se incluyen dentro de cualquier proceso de reconstrucción de volúmenes perdidos que afectan a los materiales arqueológicos es garantizar que el material añadido pueda ser eliminado o sustituido fácilmente sin provocar riesgos al original. Especialmente en este tipo de patrimonio, donde el respeto hacia el original conservado debe ser máximo en pos del valor de autenticidad del propio material. Quizá por ello, la primera reflexión que el profesional de la conservación-restauración debería hacerse a la hora de abordar una operación de reintegración, sería si realmente es necesario llevarla a cabo.

Cuándo reconstruir y por qué hacerlo son dos cuestiones básicas a las que sin duda tendremos que dar una respuesta razonada de forma objetiva. Teóricamente podremos justificarlo si con ello favorecemos la lectura y la plena comprensión de la pieza como documento histórico, entendiendo la 
laguna como una interrupción de la continuidad formal o de la decoración representada. O también en aquellos casos donde la propia estabilidad estructural de la pieza no quede garantizada. Pero en ningún caso podríamos admitir reintegraciones hipotéticas donde la legitimidad del objeto arqueológico quedase cuestionada, ni debemos tampoco intervenir cuando la escasa cantidad de material conservado no sea suficiente para permitir su reconstrucción sin caer en un falso, minusvalorando el principio de respeto hacia el original.

El criterio de mínima intervención aplicado a la conservación de nuestro patrimonio arqueológico debería de imponerse de forma más efectiva y aplicarse en todos aquellos casos donde la comprensión del objeto o su estabilidad no queden menoscabadas [Figura 1]. Nuestra experiencia laboral nos dicta que son muchas las ocasiones en donde no se precisa de un relleno de lagunas para que la legibilidad de la obra sea correcta, más aun en la era en la que estamos inmersos, donde las alternativas de reconstrucción digital son numerosas (Escriva y Madrid 2009-2010; Kotoula 2011). Quizás el problema esté más en nuestra propia mentalidad, habituada por una tradición artística de peso a que nos faciliten el trabajo visual con una intervención directa. Hoy en día, desde nuestra profesión se comprende que actuar desde el respeto al principio de mínima intervención no significa estar anulando la capacidad de la correcta difusión y comprensión de una obra, sino que implica ser consciente del daño que cualquier tratamiento directo supone para la pieza y buscar alternativas que lo aminoren o lo eviten. Nuestra responsabilidad ahora es hacer partícipes de estas ideas a otros profesionales en particular y a la sociedad en general, en definitiva a aquéllos que son cómplices de nuestras decisiones y espectadores de las colecciones.
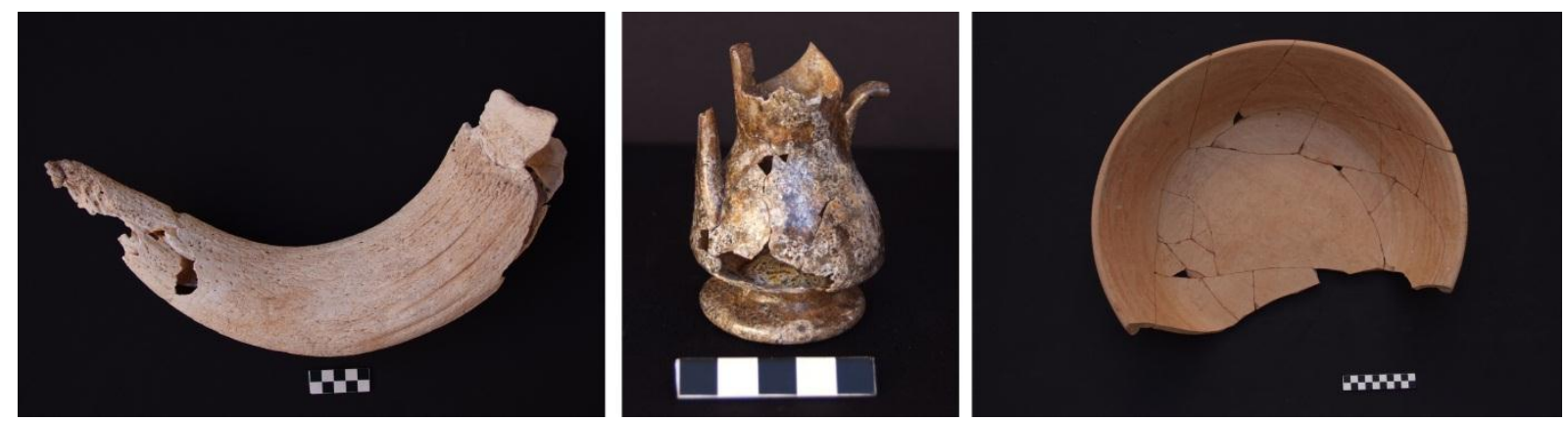

Figura 1. La alternativa de la no-reintegración en materiales de procedencia arqueológica.

Estas son algunas de las reflexiones que desde hace tiempo nos hacemos a la hora de abordar los procesos de reintegración de materiales arqueológicos en el laboratorio de restauración del Museo de Prehistoria de Valencia. En primer lugar no actuamos de forma protocolaria, sino que valoramos la conveniencia del proceso en función de las características de la obra (materia, porcentaje conservado), de la función o ubicación de la misma (difusión, investigación, vitrina, almacén) o de su estado de conservación. Así, en muchas ocasiones optamos de hecho por la alternativa de la noreintegración, aunque somos conscientes que existen situaciones en las que este proceso no es solo recomendable, sino necesario. Es en estos casos, en los que la decisión de la reintegración está plenamente justificada, donde intentamos plantear soluciones que logren aunar respeto y legibilidad de la obra. 


\section{La alternativa de las reconstrucciones desmontables}

La lista de productos y metodologías aplicadas al proceso de reintegración es muy variada, y depende no solo del tipo de material arqueológico que tengamos que intervenir, ya sea cerámica, vidrio, metal, hueso, mosaico, etc., sino del criterio profesional del propio restaurador, que obviamente estará mediatizado por su formación, su experiencia, su concepto ético, su visión de la estética o sus habilidades técnicas, entre otros aspectos.

Materiales como las escayolas dentales, empleadas para el relleno de faltantes en piezas cerámicas, las resinas epoxídicas usadas para el tratamiento de objetos de metal o vidrio, los estucos a base de ceras o las masillas sintéticas en la intervención sobre restos óseos o paleontológicos y los morteros naturales para el relleno de lagunas en pavimentos de mosaico, son solo algunos de los productos utilizados tradicionalmente en las operaciones de reintegración de materiales arqueológicos.

Dejando aparte consideraciones éticas o estéticas, cualquier profesional que decide llevar a cabo una reconstrucción de este tipo lo hace siendo consciente de las dificultades técnicas que se deberán superar y del riesgo al que el original estará sometido a lo largo de todo el proceso. Y aunque tendamos a usar de nuevo aquí el término "fácilmente reversible" para justificar nuestras intervenciones, sabemos que se trata más de un supuesto que de una realidad. Pensemos en el trabajo y en el tiempo que nos costaría eliminar todos estos morteros, estucos o masillas añadidos a las piezas, ya sea con medios mecánicos o mediante la aplicación de calor o de disolventes específicos, incluso cuando se coloca algún estrato intermedio. Y reflexionemos igualmente sobre el riesgo que esta nueva intervención supondría para la integridad del objeto y sobre las posibles alteraciones que le podríamos ocasionar.

La alternativa de las reintegraciones desmontables minimiza precisamente los principales inconvenientes a los que va asociado un proceso de reconstrucción tradicional, sin por ello dejar de cumplir su objetivo. Las ventajas que ofrece este sistema son varias:

- El nuevo material estará claramente separado del original.

- Se podrá trabajar fuera del objeto, descartando así los riesgos que se pueden ocasionar durante los procesos de lijado o acabado, aplicación del color, etc.

- Si en un futuro se decide su eliminación por cambios de criterio o envejecimiento del material empleado, éste puede ser fácilmente desmontado sin afectar al original y sustituido por otro si se considera oportuno.

Se cumple así con bastantes garantías lo que algunos autores consideran el nivel más exacto de reversibilidad, es decir, "un tratamiento que cuando es eliminado no deja señales de contaminación del mismo y ninguna alteración de la estructura del objeto que puedan ser determinadas por medios analíticos" (Fernández 2000: 74).

A pesar de las ventajas que este tipo de alternativa nos ofrece, entre los profesionales no es precisamente la metodología de trabajo más habitual y son pocos los autores que han hecho referencia en sus publicaciones a propuestas de este tipo. Quizás las más conocidas sean las intervenciones realizadas sobre materiales vítreos, debido a la complicación técnica y el riesgo potencial que implica un proceso de reintegración tradicional con resinas epoxídicas. Estos ejemplos se basan en el empleo de láminas de resinas sintéticas (acrílicas o epoxídicas) que se pegan al objeto como si fueran un fragmento más (Hogan 1993; Davison 1998; Davison 2003; Koob 2006). En ocasiones se fa- 
brican a partir de un molde sacado de un primer relleno realizado con escayola sobre la pieza (Risser 1997; Koob 2000). También en algunos trabajos puntuales sobre cerámica arqueológica encontramos reintegraciones desmontables realizadas con escayola (Koob 1987) o con nuevos fragmentos de cerámica (Hogan et. al. 1996; Geschke 2004). Otras veces, especialmente en los casos donde se conserva poco porcentaje de original, lo que se construye es un soporte con la forma del objeto sobre el cual se sujetan o adhieren los fragmentos originales de las piezas (Font 2005; Carrascosa y Lastras 2008; Quiñones y García 2009).

En el caso de los mosaicos se conocen también algunas propuestas de reconstrucciones desmontables realizadas sobre un nuevo soporte (placas de pizarra o paneles estratificados), encima del cual se colocan nuevas teselas o pseudo-teselas que reproducen la decoración perdida (Giusti et. al. 1987: 69; Gambogi, Fontanelli y Tuccino 1998; Pellegrino, Longo y Ventura 2004). Y como alternativa al empleo de morteros naturales o de otros sistemas de reintegración, está también documentado el uso de gravillas sueltas como material de relleno de lagunas en mosaicos, ya sea en piezas expuestas en museo como conservadas in situ, pudiendo seleccionar entre los diversos colores y granulometrías que nos ofrecen (Ungaro, Milella y Vitti 2000: 345-346). En pintura mural se han planteado igualmente sistemas de reintegración con arenas sueltas previamente coloreadas (Andino y Deu 2004). Se trata, en definitiva, de plantear propuestas que logren aunar respeto, reversibilidad y compatibilidad, en pos de la mejor conservación de las obras.

Desde el laboratorio de restauración del Museo de Prehistoria de Valencia intentamos aplicar estos conceptos a la hora de abordar un proceso de reintegración sobre materiales arqueológicos. Relataremos a continuación nuestra experiencia práctica sobre cuatro de las piezas pertenecientes a la colección del museo, en las que hemos recurrido al empleo de sistemas desmontables.

\section{Lebrillo cerámico (Valencia)}

El lebrillo cerámico de época islámica fue depositado en el museo en 1999, procedente de la excavación de las calles San Vicente $n^{\circ} 26$ y Mesón de Teruel 32, de Valencia. Muestra decoración sencilla en el ala de líneas cortas y gruesas a modo de pincelada. Los fragmentos de la pieza, un total de 10, habían sido pegados por el equipo de arqueólogos tras la excavación, empleando nitrato de celulosa. En primer lugar procedimos al desmontaje y limpieza del antiguo adhesivo con impregnaciones de acetona. La cerámica presentaba además adheridas concreciones de tipo calcáreo que fueron eliminadas mediante tratamiento químico (disolución al $5 \%$ de una mezcla a partes iguales de EDTA bisódico y tetrasódico).

Tras la sucesiva neutralización en baños de agua desmineralizada, el siguiente proceso era obviamente el montaje de la pieza. Los 10 fragmentos originales conservados se unieron en tres grupos independientes, empleando resina vinílica sólida K60 al 25\% en alcohol etílico. Sin embargo, en un primer momento no se aplicó ningún adhesivo para unir estos tres grupos entre sí y cerrar el conjunto, ya que para facilitar la posterior separación de los nuevos rellenos era imprescindible que las fases de reconstrucción y reintegración se realizasen de forma paralela. De no llevar a la par estos dos procesos, sería imposible hacer desmontables los materiales aplicados para completar algunas lagunas ubicadas en zonas interiores de la pieza o con formas angulosas. Por tanto, el siguiente paso fue colocar en su posición original los tres bloques de fragmentos que conformaban la pieza, pero simplemente sujetos en el interior y en el exterior de la misma con tiras de cinta de papel, de modo que posteriormente lograran separarse con facilidad. Podíamos así observar que eran dos las zonas de pérdida de materia original, una pequeña y otra de mayor tamaño, ambas en la parte superior de la pieza. El proceso de reintegración en este caso era posible y deseable porque la pieza conservaba un gran porcentaje de original, disponía de una buena parte del ala para poder ob- 
tener el molde $y$, además, iba a formar parte de un conjunto de piezas medievales seleccionadas con fines de difusión.

Para lograr aislar completamente del original el nuevo material de relleno y posibilitar su posterior desmontaje, se colocó en la zona de unión una capa de látex para moldes y, tras el secado, se procedió con las operaciones de reintegración a la manera habitual. Los bordes de las lagunas se protegieron con cinta de papel, las improntas fueron obtenidas con placas de cera y el estuco empleado, en este caso escayola dental, fue vertido con jeringuilla por unos orificios abiertos en la parte superior. Completado el proceso tras el fraguado, se eliminaron los moldes de cera y se quitaron los precintos que mantenían unidos los tres bloques de la pieza, gracias a lo cual pudimos separar sin dificultad los nuevos rellenos de escayola [Figura 2]. Se habían obtenido de este modo unas reconstrucciones completamente desmontables que, a continuación, nos permitirían continuar con el resto de procesos fuera del original. Tanto el lijado como el acabado cromático, realizado con colores acrílicos aplicados con aerógrafo, se llevaron a cabo de manera totalmente independiente a la pieza, minimizando los riesgos sobre la cerámica. Finalmente se pudo completar, esta vez sí de forma definitiva, el montaje de la pieza, incluyendo en esta ocasión los nuevos fragmentos obtenidos y ya preparados [Figura 3]. Para pegarlos al original se optó por un sistema de adhesión por puntos, colocando simplemente varios puntos de resina K60 a lo largo de la línea de unión, lo que sin duda facilitaría su desmontaje si en un futuro se decidiese su eliminación, incluso simplemente con la aplicación de un poco de calor, una de las ventajas que ofrece el uso de esta resina [Figura 4].

\section{Metapodio central de rinoceronte (Xàtiva)}

Metapodio de rinoceronte de estepa descubierto en el yacimiento del Paleolítico Medio de Cova Negra (Xàtiva) (Pérez 1977: 27-33, lám. 3-C). La pieza se encontraba expuesta en una de las salas permanentes del museo junto a los otros dos huesos que conforman la parte distal de una de las extremidades del rinoceronte, especie abundante durante el periodo prehistórico, pero de la cual conservamos pocos restos materiales. Los tres metapodios se encuentran afectados por el fuego, pero solo el central conserva dos fragmentos que unen por un punto, posibilitando así la reconstrucción del cuerpo o diáfisis del hueso y favoreciendo de este modo la comprensión del conjunto en la posición anatómica correcta. Presentaba una cortical espesa y muy resistente, lo que permitió la correcta conservación del material a pesar de que el proceso de combustión debió ser bastante completo, ya que no solo la zona compacta había adquirido de forma uniforme un color gris, sino incluso toda la zona esponjosa interior.

En una antigua intervención de restauración se había llevado a cabo la reintegración de esta zona central con estuco de escayola, que no solo presentaba un aspecto estético inadecuado, sino que había afectado al tejido óseo esponjoso de forma irreversible. Tras eliminar cuidadosamente el estuco de escayola hasta donde consideramos necesario y los restos de la plastilina que aun permanecían en el interior, planteamos una nueva propuesta de reintegración reversible. La alternativa fue la fabricación de un relleno desmontable que fue realizado en este caso con masilla epoxídica con cargas de inertes ligeros (Balsite), modelada directamente sobre el original previamente protegido con látex para moldes o papel de aluminio, según tocara zona compacta o esponjosa [Figura 5]. Tras el secado de la resina se obtenía el nuevo fragmento, que pudo separarse fácilmente del original y ser trabajado aparte para conseguir un perfecto acabado, incluyendo el proceso de retoque cromático, que fue aplicado con aerógrafo y colores acrílicos en estarcido. El paso final consistió en la fijación de la nueva pieza sobre el original, gracias a la aplicación de unos simples puntos de adhesivo Paraloid B72 al 25\% sobre la parte compacta del hueso [Figura 6]. 

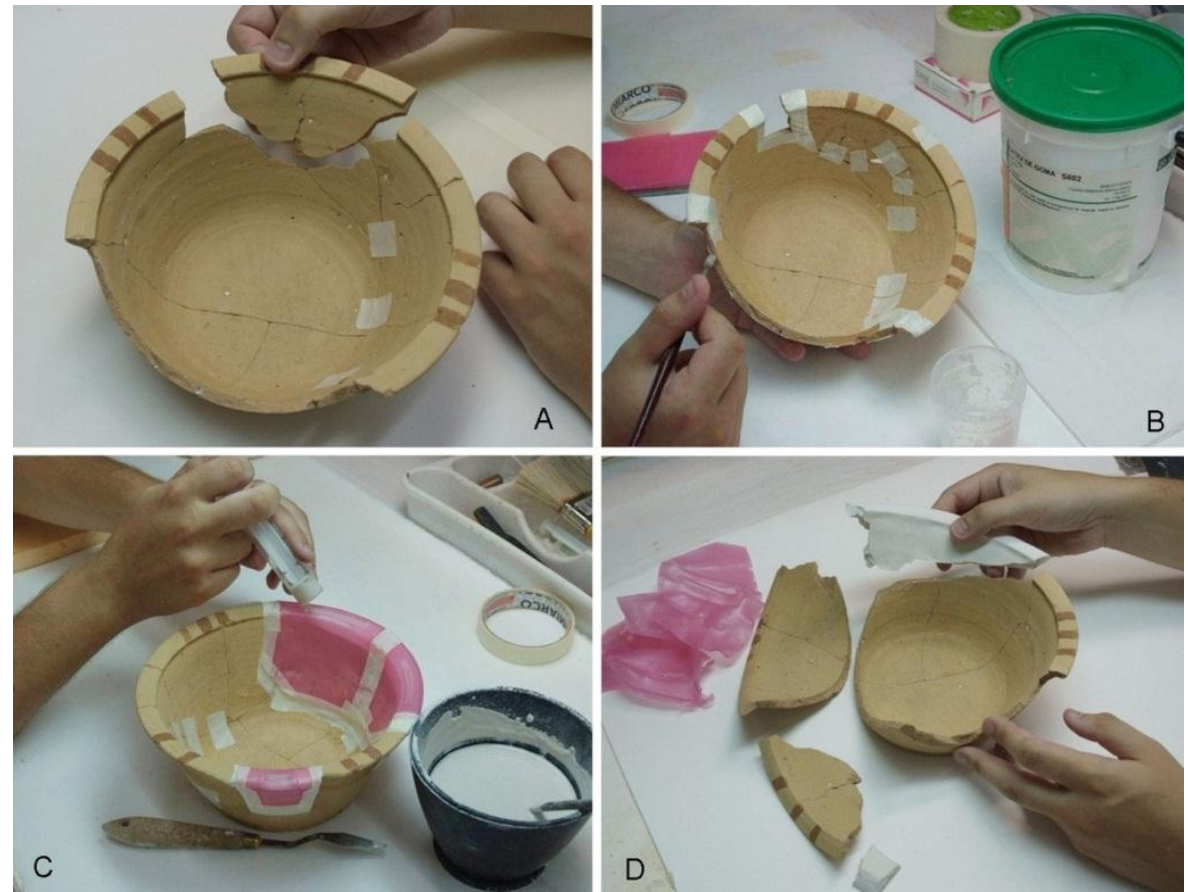

Figura 2. Lebrillo cerámico. Fases del proceso de construcción de las reintegraciones desmontables: A. Montaje provisional en grupos. B. Colocación de la capa intermedia de látex. C. Vertido de la escayola sobre los moldes de cera. D. Separación de las reintegraciones tras el fraguado de la escayola.

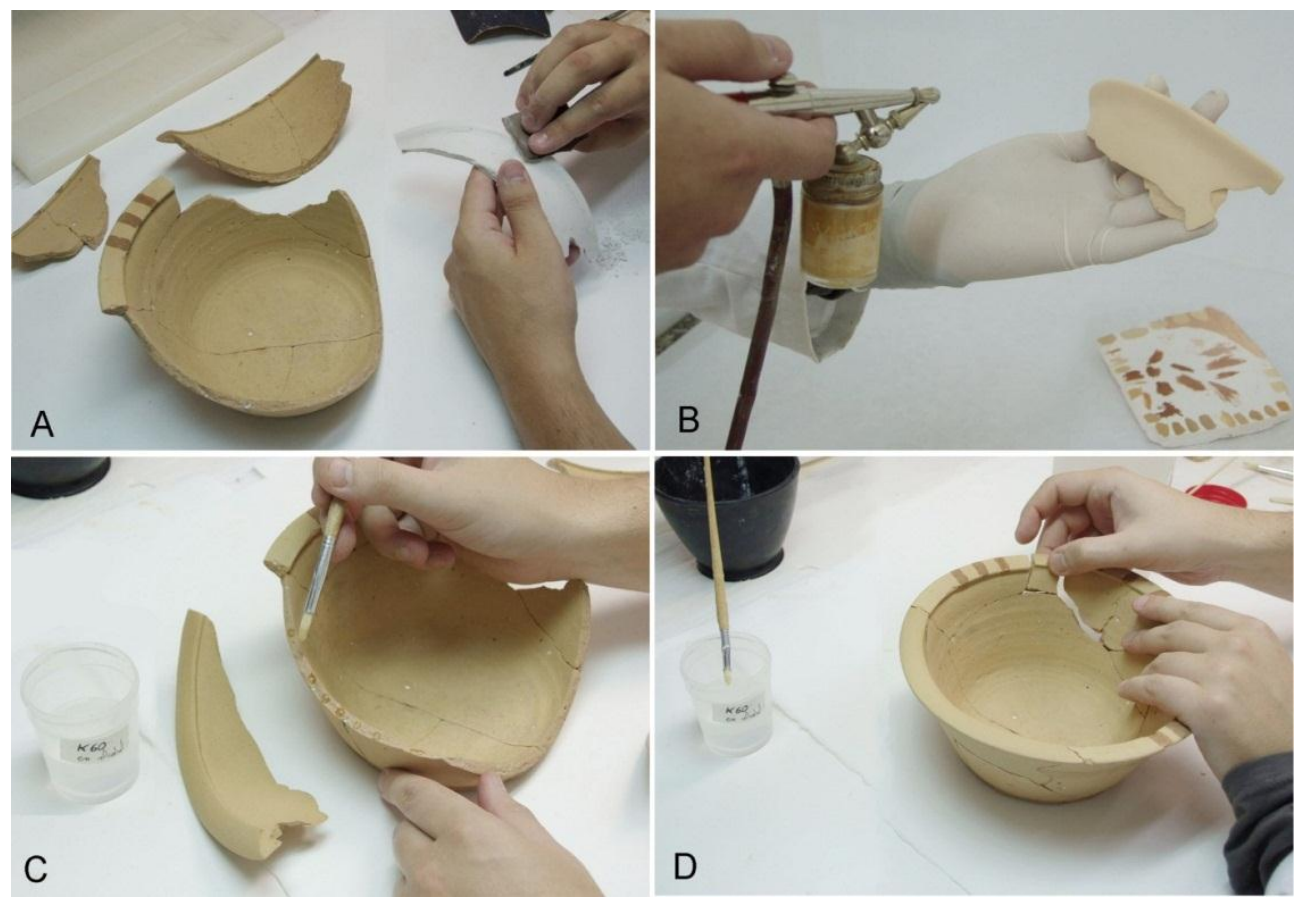

Figura 3. Lebrillo cerámico. A. Lijado de la escayola. B. Aplicación del color con la técnica del aerógrafo. C. Colocación de puntos de adhesivo para unir una de las reintegraciones. D. Montaje de otra de las nuevas piezas junto a algunos fragmentos originales. 


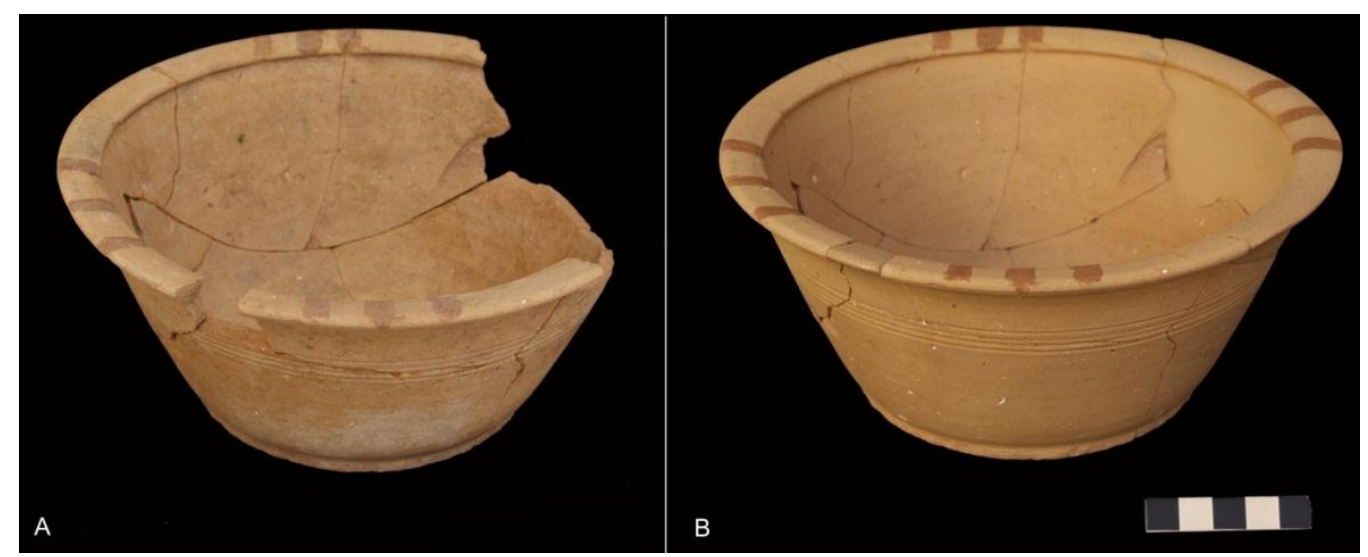

Figura 4. Lebrillo cerámico. A. Fotografía antes de la intervención B. Fotografía después de la intervención.
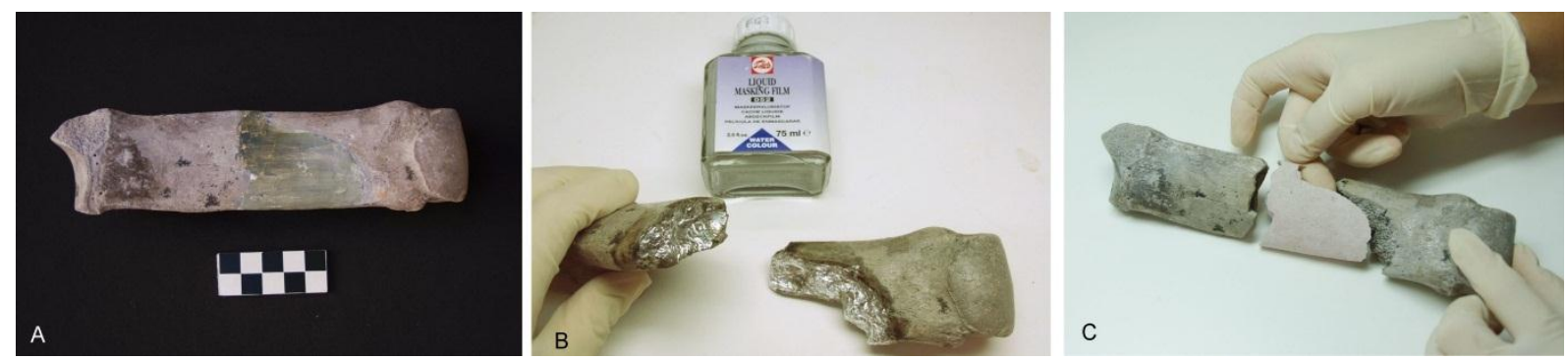

Figura 5. Metapodio central de rinoceronte. A. Fotografía de la pieza antes de la intervención, donde observamos la antigua reintegración de escayola pintada. B. Protección de las superficies de contacto. C. Realización de la reintegración desmontable con masilla sintética Balsite.

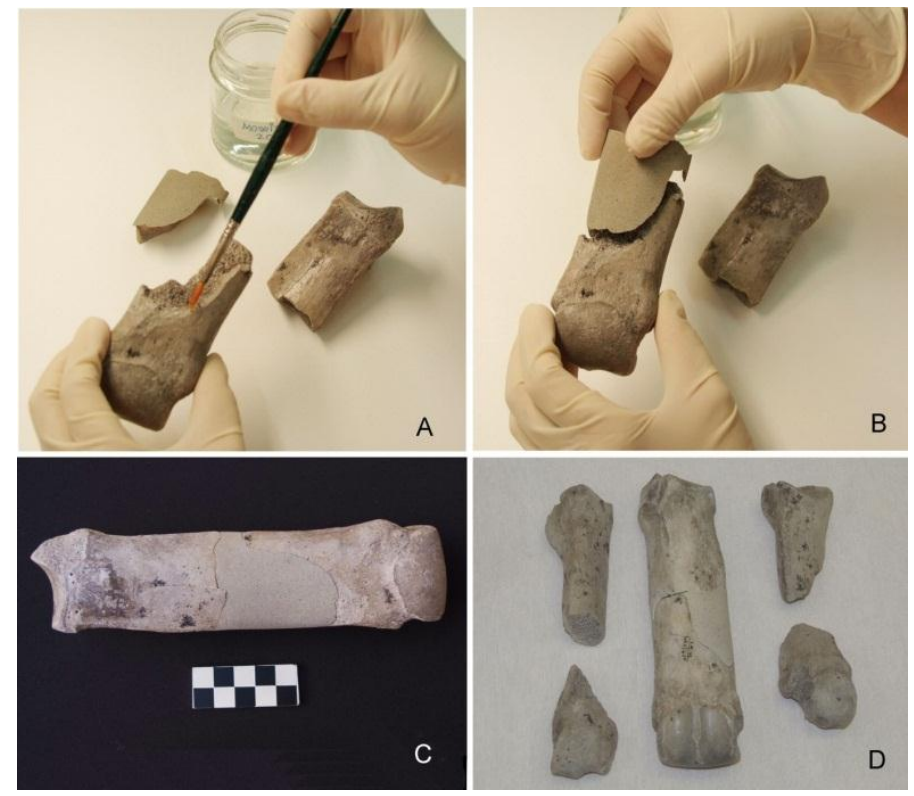

Figura 6. Metapodio central de rinoceronte. A. Colocación de puntos de adhesivo en la zona compacta. B. Adhesión de la reintegración desmontable. C. El metapodio después de la intervención. D. Conjunto anatómico de los huesos del rinoceronte expuestos en sala. 


\section{Cáliz de vidrio (Gandía)}

Copa de vidrio trabajado de época medieval (s. XV) perteneciente a uno de los ajuares de enterramiento hallados en la Colegiata de Santa María de Gandía (Vidal y Muñoz 2007). El cáliz llegó al taller de restauración con un montaje previo realizado con cintas adhesivas de papel, las cuales fueron poco a poco eliminadas con impregnaciones de acetona. Presentaba un delicado estado de conservación; un avanzado proceso de corrosión con picaduras y evidentes irisaciones en toda la superficie. La pieza estaba bastante completa, si exceptuamos pequeñas lagunas y una de mayor tamaño localizada en la zona del borde. Posiblemente en otra situación no hubiera sido necesario realizar la reintegración de los volúmenes perdidos para facilitar la lectura de la pieza, máxime teniendo en cuenta su estado de conservación y las dificultades técnicas que implica un proceso de reconstrucción en vidrio arqueológico. Pero en este caso había que adoptar otra decisión, porque precisamente en la laguna de mayor tamaño era necesario colocar algún tipo de refuerzo estructural para poder sujetar uno de los fragmentos de la pieza original que unía solo por un punto. Se trababa entonces de una operación imprescindible, no por una cuestión estética sino por motivos de estabilidad de la propia obra.

Para la realización de la reintegración desmontable empleamos en este caso una lámina de polipropileno translúcido (PP) de $0^{\prime} 5 \mathrm{~mm}$. El PP es un material químicamente inerte, apto para su uso en conservación, aunque es susceptible a los efectos de la luz solar. Se manipula y trabaja con facilidad, su apariencia es similar a la del vidrio arqueológico, y puede moldearse aplicando calor. Puede colorearse ligeramente con pigmentos disueltos en Paraloid B72 en etil acetato para lograr una buena armonía con el original. El método de trabajo es sencillo y puede repetirse tantas veces como sea necesario para corregir errores hasta conseguir el perfil deseado, sin por ello afectar a la pieza; el primer paso consiste en colocar en la parte interior del original un trozo de PP y dibujar con un perfilador el contorno de la laguna para, posteriormente, cortarlo aparte con la ayuda de un bisturí [Figura 7]. Una vez obtenida la forma la ajustamos a la pieza, reblandeciendo ligeramente la lámina con un poco de aire caliente para obtener la curvatura deseada. Para finalizar solo queda adherir la nueva pieza en el interior de la laguna con Paraloid B72 al 25\% [Figura 8].

Este sistema de reintegración desmontable con láminas sintéticas fue experimentado con éxito en otras piezas vítreas pertenecientes al mismo conjunto (Martínez, Pasíes y Peiró 2011; Pasíes, Martínez y Peiró 2012). Sus ventajas son varias, ya que permite una interacción mínima con el original durante el proceso de realización de la lámina y garantiza su fácil reversibilidad en el caso de que se desee su sustitución, ya sea por un cambio de criterio o porque se aprecie algún deterioro en la misma. El coste es mínimo y aunque requiere de una precisión a la hora del ajuste de la lámina, cualquier error no supone ningún problema, porque simplemente se desecha el nuevo fragmento y se realiza otro sin provocar daños sobre el original. Sin embargo esta alternativa ofrece también algunas limitaciones, ya que no puede ser empleada si se trata de lagunas muy grandes, con espesores de vidrio considerables o en formas de curvas muy pronunciadas.

\section{Pavimento de mosaico opus tessellatum (Benifaió)}

En 2001, en el transcurso de las obras de canalización del gas llevadas a cabo en el yacimiento de Font de Mussa, se descubrió durante el seguimiento arqueológico un pavimento musivo en opus tessellatum datado hacia final del siglo II d.C. y principio del III d.C., con medallón central policromo, que representa el tema de los orígenes de Roma y que ocupaba una habitación de aproximadamente $21 \mathrm{~m}^{2}$. Desgraciadamente el pavimento apareció completamente partido en dos debido a la construcción de un colector en fechas que no hemos podido determinar con certeza, actuación que obviamente se llevó a cabo sin la correspondiente vigilancia arqueológica. 
Las excepcionales circunstancias que caracterizaron el hallazgo imposibilitaron su conservación in situ, y la pieza tuvo que ser extraída y trasladada en secciones al laboratorio de restauración del Museo de Prehistoria, donde se desarrollaron las operaciones de limpieza, colocación de nuevo soporte y exhibición en una de las salas permanentes del museo (Pasíes y Buendía 2004). Las diferentes planchas que conformaban el conjunto fueron reagrupadas encima de un nuevo entarimado construido a medida sobre el suelo. El principal problema era buscar una solución a la gran laguna provocada por el colector y que recorría de parte a parte el mosaico. En ningún momento pretendimos ocultar su presencia con algún tipo de reconstrucción realizada con nuevas teselas o con un mortero inciso dibujando la forma de las mismas y posteriormente coloreado, sistemas estos utilizados en muchas de las intervenciones que a nivel nacional e internacional se han venido realizando hasta la fecha sobre pavimentos de mosaico (Pasíes y Carrascosa 2002). Nuestra idea era no negar la evidencia del daño provocado, que por desgracia forma ya parte de la historia documentada de la pieza, sino que pudiera al menos servirnos de recordatorio de lo que nunca se debió hacer. Buscamos sin embargo un punto intermedio, y optamos por la decisión de insinuar al menos las principales líneas del diseño decorativo. Como material de relleno la opción tradicional hubiera sido la aplicación de un mortero natural a base de cal e inertes variados, pero en este caso recurrimos al empleo de gravillas, seleccionadas de acuerdo al color y la granulometría más adecuadas.
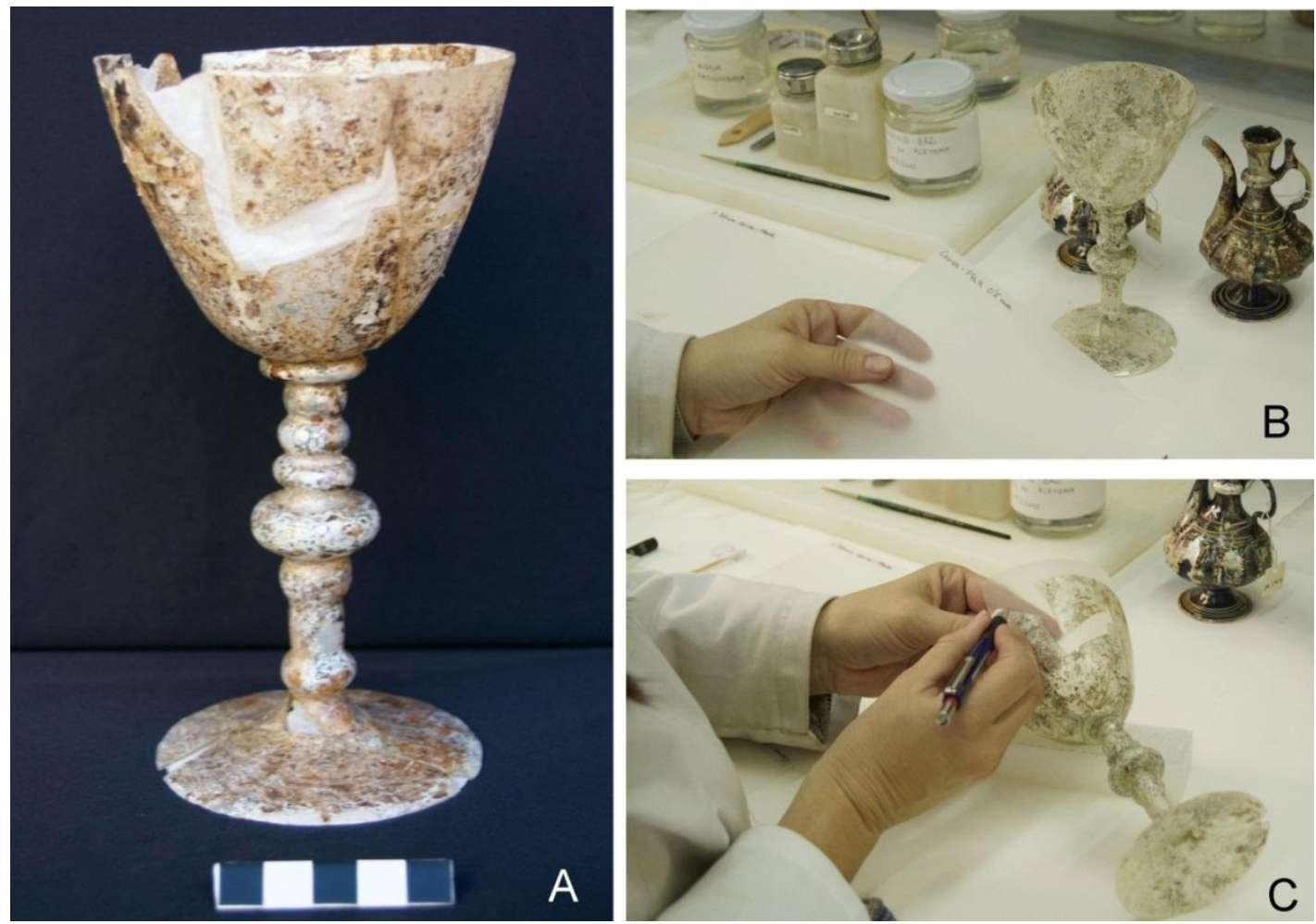

Figura 7. Cáliz de vidrio. A. Fotografía antes de la intervención, donde observamos los refuerzos de papel adheridos a la pieza. B. Selección de la lámina de PP de $0^{\prime} 5 \mathrm{~mm}$. C. Colocación de la lámina de PP en el interior de la laguna y perfilado a mano del contorno. 

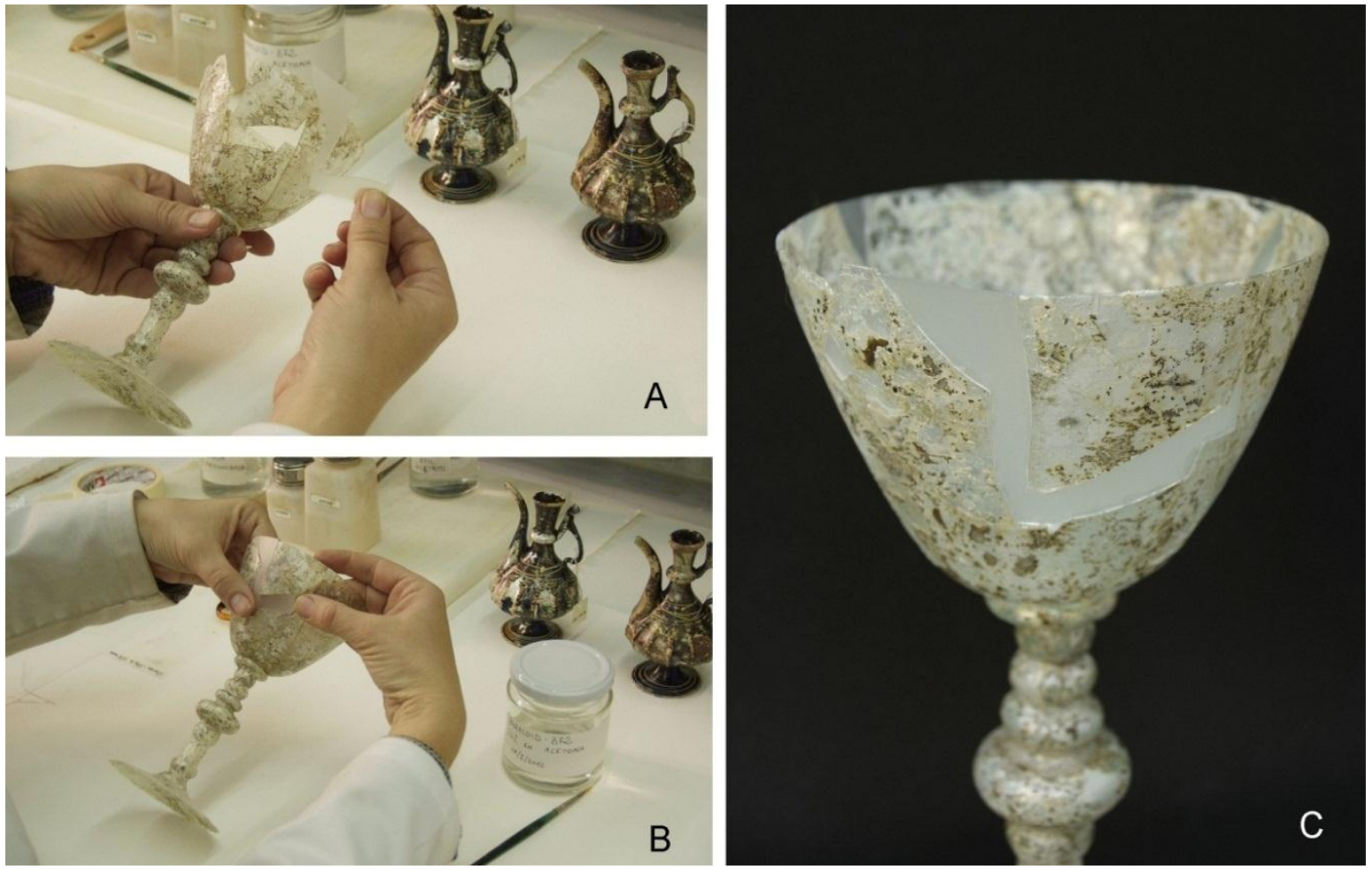

Figura 8. Cáliz de vidrio. A. El fragmento desmontable ya recortado con bisturí. B. Adhesión de la lámina de PP en el interior de la laguna con Paraloid B72. C. Detalle de la pieza con la nueva zona reconstruida.

El primer paso fue la sujeción a la estructura de unos finos listones de madera cortados a medida, que se adaptaron a las formas del dibujo decorativo central que deseábamos completar [Figura 9]. Sobre estos se colocó gravilla de color negro adherida con resina epoxídica, convirtiéndose en los únicos puntos fijos de todo el sistema. Definidos así los espacios, solo quedaba completar el relleno de la laguna colocando en el resto de la superficie una mezcla de gravillas sueltas de color negro y marfil en una proporción 1:7, que ofrecía una vibración, textura y tonalidad muy armónica con el original. El mismo material fue colocado en otras lagunas más pequeñas que también presentaba el pavimento. Una de ellas correspondía a una zona de quemado donde se emplearon hasta tres colores diferentes de gravillas para entonarlo con el color de la piedra original. En cualquier caso es una alternativa rica de posibilidades, que permite obtener resultados estéticos muy buenos con recursos muy sencillos [Figura 10].
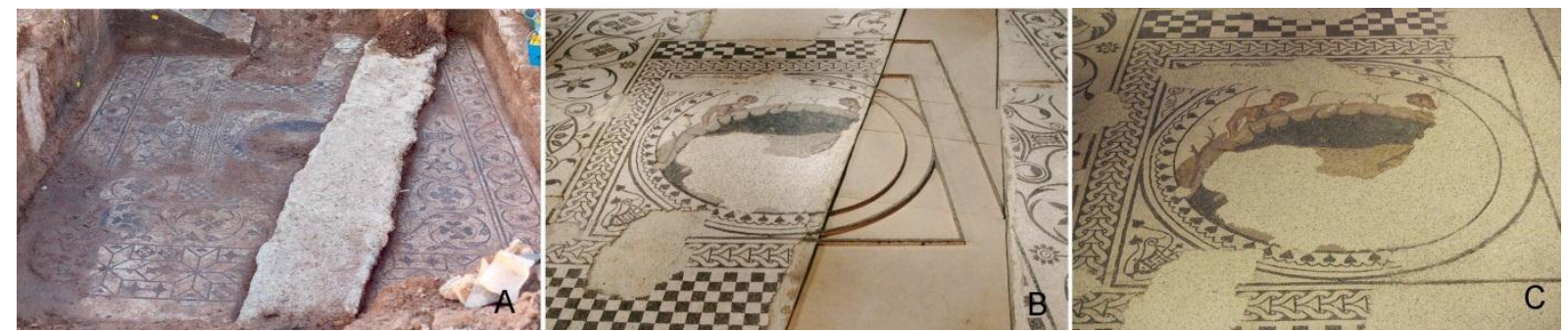

Figura 9. Pavimento de mosaico. A. El mosaico in situ antes de proceder a su extracción. B. Tras su restauración el pavimento se ubica en una de las salas del museo. Colocación de los listones con gravilla negra adherida para dibujar las principales líneas decorativas perdidas. C. Detalle de la zona central tras colocar la gravilla suelta. 


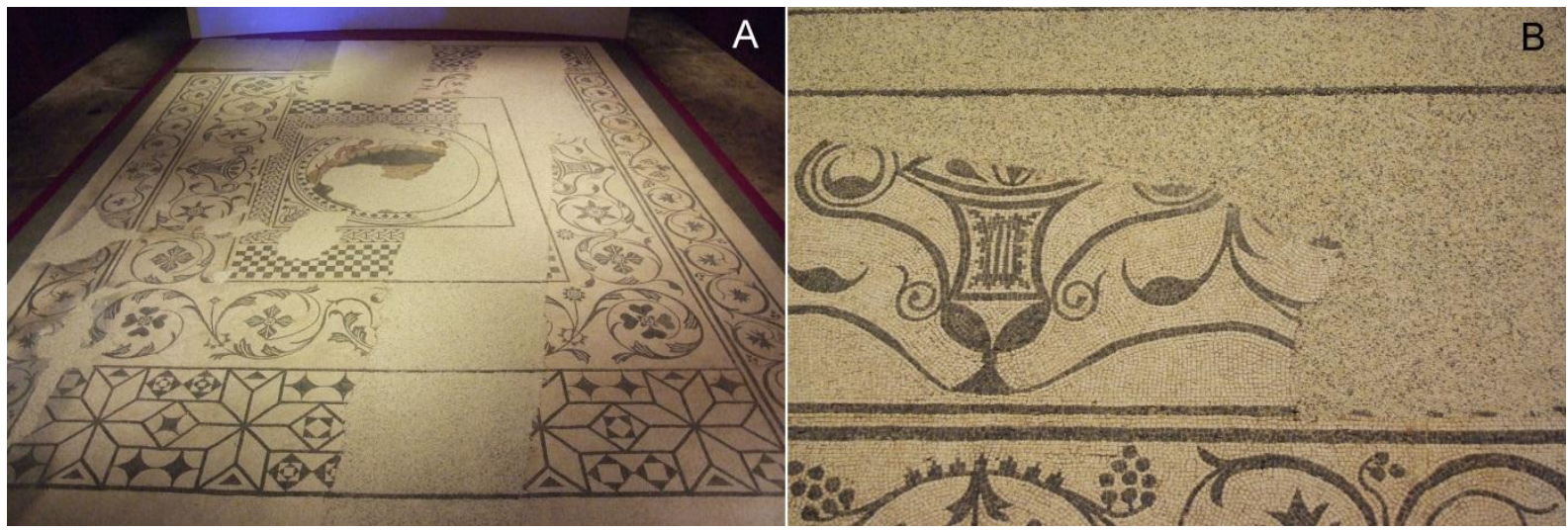

Figura 10. Pavimento de mosaico. A. Fotografía final del pavimento en una de las salas de exposición del Museo de Prehistoria de Valencia. B. Detalle de una zona del pavimento donde se aprecia la reintegración reversible de lagunas realizada con una combinación de gravillas sueltas

Podemos de nuevo confirmar que las ventajas que obtenemos con el empleo de este sistema son numerosas. Se trata de una propuesta técnicamente muy simple, limpia, rápida de ejecutar, totalmente reversible, económica y compatible con los materiales originales. Desde nuestro punto de vista es ideal para realizar la reintegración de pavimentos de mosaico conservados en museo, más aun si se trata de piezas que puedan ser trasladadas para ser expuestas en diversas exposiciones temporales. Indudablemente las obras deben presentarse en horizontal, pero es obvio que el mejor modo de conservar un pavimento de mosaico sin caer en una grave descontextualización es exponerlo sobre el suelo, y no colgado de una pared. Se requiere solamente de la preceptiva vigilancia de la sala y de unas sencillas labores de mantenimiento en el caso de que alguna persona haya podido remover la gravilla.

Pero no solo es un sistema óptimo para mosaicos expuestos en museos, sino incluso para piezas conservadas in situ. De hecho hemos experimentado que puede ser una buena alternativa para rellenar lagunas en las que se ha perdido la superficie teselar, pero donde se conservan el resto de estratos preparatorios, colocando en este caso algún tejido intermedio, lo que permitiría un eficaz estudio técnico del pavimento (Pasies y Mai 2010).

\section{Conclusiones}

Cualquier proceso de reintegración supone una intervención directa sobre las piezas que no estará exenta de debates éticos sobre su necesidad, de decisiones sobre la metodología que se empleará, ni de la posibilidad de ocasionar daños al original. En el caso del tratamiento de materiales arqueológicos, donde la exigencia del respeto a su autenticidad es una característica que el conservadorrestaurador debe priorizar, la viabilidad y las soluciones adoptadas deben ser bien reflexionadas.

Desde nuestra perspectiva actual existen recursos suficientes para que los criterios de reversibilidad y mínima intervención puedan convivir perfectamente dentro de un proceso de reintegración. Actuar con esa mentalidad no implica que no haya que intervenir, ni que no podamos buscar alternativas que logren aunar respeto y legibilidad de la obra. Es ahí donde nuestra investigación se centra en el planteamiento de propuestas que cumplan estos objetivos y que a la vez no precisen de una complicada metodología de elaboración ni de grandes dificultades técnicas para llevarlas a cabo. 
La alternativa de las reintegraciones desmontables supone desde nuestro punto de vista un ventajoso avance en el cumplimiento de los criterios básicos de la restauración. Como hemos comprobado podemos recurrir al empleo de productos diversos dependiendo del material con el que trabajemos, pero los objetivos y méritos del sistema son siempre los mismos: crear rellenos desmontables, claramente separados del original, lo que posibilita su fácil reversibilidad sin por ello perjudicar la adecuada lectura de la obra. A nivel técnico las ventajas son varias y la complejidad del proceso de reintegración disminuye notablemente con respecto a los métodos tradicionales, ya que, al trabajarse los añadidos fuera del original, se agilizan los procesos de acabado y no deben preocuparnos en exceso los posibles errores que se puedan provocar, ya que estos no afectarían al material original y podrían subsanarse sin problemas. Se trata además de una metodología reversible, donde se minimiza la manipulación de la pieza y con ello los riesgos de provocar algún daño sobre la obra, y no solo durante el proceso de realización de la propia reintegración, sino incluso en una hipotética eliminación posterior de la misma.

En cualquier caso apostamos por aquellas intervenciones que continúen esta línea de investigación, donde el principal objetivo sea el desarrollo de sistemas de reintegración fácilmente reversibles en los que no se ponga en riesgo a las piezas. Y no se trata solo de una cuestión de criterios, sino de optimización de recursos, en la búsqueda de propuestas sencillas, económicas y efectivas, que ofrezcan un resultado óptimo sin afectar a la correcta conservación del material original.

\section{Agradecimientos}

Cuando entre los profesionales que trabajan en un museo existe un ambiente de colaboración interdisciplinaria, con una mentalidad abierta a nuevos planteamientos que sean beneficiosos para la conservación de las piezas, el trabajo del conservador-restaurador se hace mucho más sencillo. Nuestras palabras de agradecimiento a la directora del Museo de Prehistoria de Valencia, Helena Bonet, por su apoyo y confianza, a Milagros Buendía, $M^{a}$ Amparo Peiró, Isabel Ferri y Manuel Moragues, que participaron en la realización de algunos de estos trabajos, y a todos los conservadores con los que diariamente tenemos la oportunidad de colaborar. Igualmente al Archivo del propio museo, de donde hemos extraído toda la documentación fotográfica mostrada en este artículo.

\section{Bibliografía}

ANDINO, L. y DEU, N. (2004). "Una alternativa a la reintegración tradicional. El uso de arenas coloreadas", Kausis, 2: 33-35. http://www.patrimonioculturaldearagon.com/patr/contenido/publicaciones-escuelas-taller [consulta: 12/3/2012].

APPELBAUM, B. (1987). "Criteria for treatment: reversibility", Journal of the American Institute for Conservation, 26-2: 65-73. http://cool.conservation-us.org/jaic/articles/jaic26-02-001.html [consulta: 12/3/2012].

CARRASCOSA, B. y LASTRAS, M. (2008). "Sistemas alternativos para la reintegración y musealización de cerámicas arqueológicas". En 17th Meeting on Heritage Conservation. Castellón, Vila-real, Burriana: Fundación la Llum de les Imatges, Conselleria de Cultura i Esport, Generalitat Valenciana, 509-512.

DAVISON, S. (1998). "Reversible fills for transparent and traslucent materials", Journal of the American Institute for Conservation, 37-1: 35-47. http://cool.conservation-us.org/jaic/articles/jaic37-01-004.html [consulta: 12/3/2012].

DAVISON, S. (2003). Conservation and Restoration of glass. Oxford: Butterworth-Heinemann. 
DELLA TORRE, S. (2003). "Il rispetto dell'esistente e l'irreversibilità dell'azione". En La reversibilità nel restauro. Riflessioni, esperienze, percorsi di ricerca. Atti del Convegno di Studi, Bressanone: Arcadia Ricerche, 15-22.

ESCRIVA, F. y MADRID, J.A. (2009-2010). "El mundo virtual en la restauración. Aplicaciones virtuales para la conservación y restauración del Patrimonio", Arché. UPV, Valencia, 4-5: 11-20.

FERNÁNDEZ, Ma I. (2000). "Reflexiones sobre reversibilidad: la reversibilidad entre la teoría y la praxis", $P H$, IAPH, 30: 70-74. http://www.iaph.es/Patrimonio_Historico/cd/ficheros/10/ph30-70.pdf [consulta: 12/3/2012].

FONT, L. (2005). "El Departament de conservació I restauració del Museu d'Història de la Ciutat", Quaderns tècnics de I'MHCB. Conservació i restauració, 1: 11-31.

GAMBOGI, P.; FONTANELLI, R. y TUCCINO, W. (1998). "Restauro e musealizzazione in situ del mosaico della mansio romana di Collesalvetti". En Atti del V Colloquio dell'associazione Italiana per lo Studio e la Conservazione del Mosaico AISCOM, Roma: Edizioni del Girasole, 153-160.

GIUSTI, A.M. et. al (1987). "Restauration d'une mosaïque du baptistere de Florence; propositions tecnhiques et methodologiques". En Mosaicos nº 4. Conservación in situ, Soria: Diputación Provincial de Soria, 57-84.

GESCHKE, R. (2004). “Ceramic gap-fills for ceramic restoration", The Conservator, 28: 74-83.

HOGAN, L. (1993). "An improved method of making supportive resin fills for glass", Conservation news, London, 50: 29-30.

HOGAN, L. et. al (1996). "Reconstructing major missing areas of ceramics vessels using clay". En Triennal meeting $\left(11^{\text {th }}\right)$ ICOM-CC, Edinburgh, Scotland: ICOM-CC, 833-838.

ICOM (2008). "Terminology to characterize the conservation of tangible cultural heritage". En Triennal meeting (15th), New Delhi: ICOM-CC. http://www.icom-cc.org/54/document/icom-cc-resolution-terminologyenglish/?id=744 [consulta: 13/3/2012].

KOOB, S. (2000). "New techniques for the repair and restoration of ancient glass". En Tradition and innovation: advances in conservation, London: The International Institute for Conservation of Historic and Artistic Works, 92-95.

KOOB, S. (2006). Conservation and care of glass objects. London: Archetype Publications.

KOOB, S. (1987). "Detachable plaster restorations for archaeological ceramics". En Recent advances in the conservation and analysis of artifacts. Jubilee conservation conference, London: Summer Schools Press, 63-65.

KOTOULA, E. (2011). “Old interventions \& New possibilities", e-conservation magazine. 19: 40-47. http://econservationline.com/content/view/997 [consulta: 13/3/2012].

MARTÍNEZ, B.; PASÍES, T. y PEIRÓ, Ma.A. (2011). “Reversibility and minimal intervention in the gap filling process of archaeological glass", e-conservation magazine, 20: 40-54. http://www.econservationline.com/content/view/1010 [consulta: 12/3/2012].

ODDY, A. (1999). “Does reversibility exist in conservation?". En Reversibility-does it exist?, Oddy, A. y Carroll, S. (eds). London: British Museum, 7-10.

PASÍES, T. y CARRASCOSA, B. (2002). "La reintegración de lagunas en mosaicos pavimentales". En Actas del XIV Congreso de Conservación y Restauración de Bienes Culturales, Valladolid: Excmo. Ayuntamiento de Valladolid, 717-725. http://es.scribd.com/doc/49861926/2002-Valladolid-reintegracion-mosaico [consulta: 12/3/2012]. 
PASÍES, T. y BUENDÍA, M. (2004). "Mosaico de Font de Mussa (Benifayó, Valencia): Los procesos de conservación y restauración". En El mosaico romano de "los orígenes de Roma". Barcelona: Gas Natural SDG, 101-115. http://es.scribd.com/doc/49864956/2004-Benifayo-restauracion [consulta: 12/3/2012].

PASÍES, T. y MAI, C. (2010). "Mosaico en Banyets de la Reina. III Fase". En Actuaciones arqueológicas en la provincia de Alicante 2008, CD-ROM, Alicante: Colegio oficial de Doctores y Licenciados en Filosofía y Letras y en Ciencias de Alicante. http://es.scribd.com/doc/58158443/2010-Pasies-T-y-Mai-C-CD-Alicante [consulta: $12 / 3 / 2012]$

PASÍES, T., MARTÍNEZ, B. y PEIRÓ, Ma.A. (2012). “Cuestiones de criterio en la intervención sobre vidrio arqueológico: hacer realidad el concepto de reversibilidad y mínima intervención". En Actas del V Congreso del Grupo Español del IIC. Patrimonio Cultural. Criterios de calidad en intervenciones, Madrid: Grupo Español de Conservación, 285-294.

PELLEGRINO, L., LONGO, A. y VENTURA, L. (2004). "Intervento di riconfigurazione". En Atti I Convegno Internazionale di Studio La Materia e i Segni della Storia. Apparati musivi antichi nell'area del Mediterraneo, Palermo: Dario Flaccovio editore, 677-680.

PÉREZ, M. (1977). Los mamíferos del yacimiento musteriense de Cova Negra (Játiva, Valencia). Valencia: Serie Trabajos Varios 53. Diputación de Valencia.

http://www.museuprehistoriavalencia.es/resources/files/TV/TV053_Perez_Ripoll.pdf [consulta: 12/3/2012].

QUIÑONES, M. y GARCÍA, J. (2009). "Restauración de vidrio arqueológico. Montaje de vidrio arqueológico sobre resina en las lámparas de la sinagoga de Lorca para su exposición". En XX Jornadas de Patrimonio Cultural de la Región de Murcia, Murcia: Consejería de Cultura y Turismo Región de Murcia, 267-275. http://www.arqueomurcia.com/archivos/publicaciones/jornadaspatri20/26\%20restauracion\%20vidrio\%20re sina\%20Lorca.pdf [consulta:12/3/2012].

RISSER, E. (1997). "A new tecnique for the casting of missing areas in glass restoration", Journal of Conservation \& Museum Studies, 3. http://cool.conservation-us.org/jcms/issue3/risser.html [consulta: 12/3/2012].

UNGARO. L., MILELLA, M. y VITTI, M. (2000). "Restauri ai mercati di Traiano el ai fori imperiali: tra conservazione e valorizzazione". En Actas del XIII Congreso de Conservación de Bienes Culturales, Lérida: Generalitat de Catalunya, 339-350.

VIDAL, C. y MUÑOZ, G. (2007). "La iglesia colegiata de Santa María de Gandía. Investigaciones recientes", Caesaraugusta, 78: 729-738. http://ifc.dpz.es/recursos/publicaciones/27/22/53.vidal.pdf [consulta: 12/3/2012].

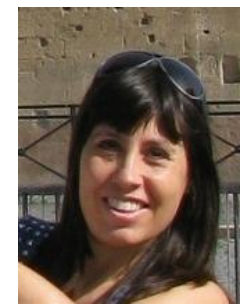

\section{Dra. Trinidad Pasíes Oviedo}

Museo de Prehistoria de Valencia.

c/ Corona 36, 46003, Valencia.

trini.pasies@dival.es

Restauradora del Museo de Prehistoria de Valencia. Doctorada en 2004 por la Universidad Politécnica de Valencia, completa su formación con estancias en varios centros internacionales. Ha dirigido numerosas intervenciones sobre materiales arqueológicos, publicando en diversas revistas científicas los resultados de sus investigaciones e impartiendo cursos de especialización en colaboración con entidades públicas y privadas

Artículo recibido el 27/03/2012

Artículo aceptado el 22/07/2012 